\section{Gabriella Buzzi Michael A. Moskowitz}

Received: 15 April 2005

Accepted in present form: 15 April 2005

Published online: 13 May 2005

M.G. Buzzi ( $\square)$

Headache Centre,

IRCCS Santa Lucia Foundation,

Via Ardeatina 306, I-00179 Rome, Italy

e-mail: mg.buzzi@hsantalucia.it

Tel.: +39-06-515011

Fax: +39-06-51501972

M.A. Moskowitz

Neuroscience Center,

Department of Radiology and Neurology,

Massachusetts General Hospital,

Harvard Medical School,

Charlestown, MA, USA

\title{
The pathophysiology of migraine: year 2005
}

\begin{abstract}
Migraine is a complex pathophysiology in which both central and peripheral components of the trigeminal pain pathway probably play a significant role, both in the symptoms and signs of the attack and in the mechanisms of action of antimigraine compounds, such as triptans, which constitute the most important therapy for aborting migraine pain and posses several mechanisms on 5-HT receptor-mediated actions. The experimental neurogenic inflammation model represents a simple procedure to obtain preliminary information on well characterized receptortargeted drugs. The apparent paradox observed with certain drugs that are shown to be effective in this model but not in clinical trials offers the opportunity to better manipulate structure-activity to obtain the best pharmacological profile using an array of experimental models. The observation that nitric oxide donors induce migraine-like pain in migraineours
\end{abstract}

and that nitric oxide plays a pivotal role in the control of several functions in the central nervous system, has prompted the use of such molecules for better understanding the pathophysiology of migraine attacks. A link between central and peripheral components of the trigeminal pain pathway is provided by the observation that cortical spreading depression in the rat activates trigeminovascular afferents and induces a series of cortical meningeal and brainstem events consistent with the development of headache. Studies in humans support the hypothesis that cortical spreading depression underlies migraine aura. Therefore, it is possible that visual, motor or sensory aura might be responsible for the generation of the pain through the above mechanisms

Key words Trigeminovascular system - Neurogenic inflammation • Nitric oxide $\cdot$ Cortical spreading depression $\cdot$ Migraine aura

\section{Introduction}

Despite the high frequency of migraineurs in the general population, the pathogenesis of this disorder is still unclear.
There is a great need for a better understanding of the mechanisms underlying the pain, the accompanying symptoms, as well as the premonitory symptoms and the aura. Progress in translational research is impeded by the lack of a validated experimental model and by the lack of tools to 
study specific sites of pharmacological activity in humans.

The role of the brain in migraine remains unclear, although recent findings link migraine to other neurological conditions, i.e., hemiplegic migraine is allelic with episodic ataxias and cerebellar disorders [1]. This observation provides a possible link between different pathological conditions residing primarily in the central nervous system (CNS).

The brain is usually considered as an insensitive organ. However, like other visceral organs, the brain possesses connective tissue coverings that serve to protect the organ [2]. In fact, the meninges receive a rich vascular supply and dense trigeminal as well as autonomic innervation, and contain resident macrophages and mast cells that participate in an inflammatory response when the brain or the meninges themselves are chemically or otherwise injured. The pattern of innervation is preserved among mammals, no matter what the size and specialisation of the brain, and it is unilateral, except for the midline, the latter being the possible explanation for the bilateral location or sideshifting of head pain during an attack.

\section{The trigeminovascular system}

During the last two decades the trigeminovascular system (TVS) has been proposed [3] and studied [4-6] to evaluate the peripheral component of the migraine attack and to possibly identify the mechanism of action of migraineaborting drugs, namely ergot derivatives and triptans. The TVS has been challenged in the rat with chemical stimulation (systemic capsaicin) or unilateral electrical trigeminal ganglion stimulation (UETGS) to induce neurogenic inflammation (NI), i.e., vasodilation and plasma protein extravasation in tissue receiving trigeminal innervation (dura mater, conjunctiva, eyelid, lip) upon release of vasoactive peptides (CGRP, SP) from nerve endings [5, 6]. Degranulation of mast cells accompanies the oedema response [7] and a stimulation intensity-dependent increase of CGRP is observed during UETGS in the plasma obtained from the superior sagittal sinus (SSS) [8].

The antimigraine drugs dihydroergotamine (DHE) and sumatriptan (SUM), the first triptan synthesised for migraine attack treatment, were effective in blocking NI [4, 6-9]. Because of strong claims that these drugs had limited central penetration, a peripheral, prejunctional mechanism of action was proposed $[10,11]$. For example, SUM, given at therapeutic doses, is not able to cross the blood-brain barrier (BBB) unless the latter is altered [12]. Interestingly, in the NI model, extravasation occurs in rat dura mater but not brain, thus ruling out the possibility that electrical stimulation of the trigeminal ganglion, although potent, is able to damage the BBB [5]. An inter- esting finding is that UETGS, while inducing tissue oedema, also provokes conjunctival injection, tearing and rhinorrhoea on the stimulated side, suggesting the presence of a trigeminal-autonomic reflex. Increase of CGRP plasma levels is also reported in the cat following trigeminal ganglion stimulation, and in blood obtained from the jugular vein of humans during migraine attacks [13]. The levels of GCRP decrease in humans, and the pain improves as well, following SUM administration. This observation should be interpreted cautiously, as CGRP plasma levels increase at the very beginning of electrical stimulation of the trigeminal ganglion and start decreasing when the stimulation sustains [8]. Nevertheless, a recent clinical trial using a CGRP receptor antagonist proved that this drug was effective in aborting migraine headaches, and raising the profile of CGRP in acute attacks [14].

Interestingly, among migraine patients, about $45 \%$ of them report local autonomic signs during unilateral severe migraine attacks [15] and a larger proportion of responders to SUM, rizatriptan and zolmitriptan are found among migraine patients in whom local signs of neurovascular activation are present during attacks [16]. Gadoliniumenhanced MRI is normal during migraine attacks, thus ruling out overt damage to the BBB [17]. While dural inflammation was not observed with this procedure, there is very preliminary evidence that NI, when measured by Tc $99 \mathrm{~m}$ HAS SPECT scan [18], occurs in the dura mater during the migraine attack, and retro-orbital oedema (vascular inflammation with plasma extravasation in the basal venous vessels of the skull) has been described using the same technique during the active period in cluster headache [19].

\section{The trigeminal pain pathway and CNS-penetrating 5-HT1B/1D agonists}

The NI model has been used for several years to predict the efficacy of other 5-HT1B /1D agonists. Second-generation triptans differ from SUM in their ability to cross the BBB at therapeutic doses. Some of those agonists, such as avitriptan, show little potency to block NI but are able to block migraine pain [20]. On the contrary, the conformationally restricted SUM analogue CP122,288 is a potent inhibitor of NI, but it is not effective on migraine pain [21, 22]. It seems that the oedema component of NI is less clinically relevant than vasodilation for the acute attack. Because the migraine attack has been regarded by some as a discharge from a central "generator", probably located in the brainstem [23], a central mechanism in rostral brain stem has been advocated as a trigger. Although it may be unrelated to this trigger, the central action of the "second-generation triptans" was not more effective for treating pain or block- 
ing headache recurrence. In fact, the degree of pain relief and pain freedom, as well as recurrence, is about the same for SUM and the newly synthesised triptans.

Activation of neurons, seen as increased $c$-fos expression in the TNC following trigeminal ganglion, dural or SSS stimulation in the cat, is blocked by local application of triptans with peripheral+central actions on primary trigeminal afferents $[24,25]$. The expression of $c$-fos can be suppressed not only by drugs that block central actions within TNC, but also by drugs that block, primarily, peripheral inputs. CP122,288 does not block TNC cell activation, whereas eletriptan, which is able to inhibit both NI and $c$-fos expression in the TNC, is effective in blocking migraine pain [26]. On the basis of these observations, the TNC has been proposed as the possible site of action of CNS-penetrating compounds in migraine attack treatment. In vivo pretreatment with CP93,129, SUM or DHE inhibits $c$-fos expression in the TNC induced by subarachnoid haemorrhage [27]. $c$-fos activation following unilateral spreading depression is inhibited by both SUM and by trigeminal denervation, suggesting a role for peripheral inputs in this model and their involvement in the mechanism of action of the drugs [28]. Levy and colleagues have provided strong evidence implicating 5-HT1B/D receptor blockade of neurotransmitter release from central endings on trigeminovascular afferents within trigeminal nucleus caudalis [29].

In some patients dystonia and akathisia have been reported as well as the efficacy of SUM in treating palatal myoclonus [30-33], thus suggesting a central action of the drug. The presence of SUM binding sites is described in several CNS areas other than the TNC, and the substantia nigra also possesses SUM binding sites, providing a locus for drug activity in the above patients [34, 35]. SUM and zolmitriptan are able, when systemically administered or locally applied on brain slices, to abolish NOS and cGMP increase following NMDA receptor activation [36]. The usefulness of the NI model is also evident when testing prophylactic drugs such as valproate, a gamma-amino butyric acid (GABA)-agonist, which has been shown to be effective in blocking dural plasma extravasation following UETGS and SP administration, via a bicucullinereversible mechanism [37] and c-fos expression following intracisternal capsaicin, suggesting a role for GABA A receptor in migraine attack pathophysiology [38].

To summarise, migraine is a complex pathophysiology in which both central and peripheral components of the trigeminal pain pathway probably play a significant role, both in the symptoms and signs of the attack and in the mechanisms of action of antimigraine compounds. In fact, triptans, which constitute the most important therapy for aborting migraine pain, possess several mechanisms on 5-HT receptor-mediated actions and some of them are still not completely understood. More detailed clinical research studies are needed in order to clarify what is still unknown about these drugs. Does sumatriptan cross the BBB during a migraine attack? Drugs that are devoid of peripheral action should be designed in order to test the existence of a CNS generator. Patients with unilateral migraine pain show decreased pain perception threshold during corneal reflex recording [39]. This observation suggests sensitisation of the peripheral and/or central pain pathway [40]. From this perspective, the triptans can be regarded as inhibitors of the perpetuation of pain mediated by the peripheral inputs from the TVS.

\section{NO donors activate the trigeminal pain pathway: a link with a clinical model of migraine}

The ability of nitrovasodilators to act as prodrugs that release nitric oxide (NO) in several body tissues (vessel wall, lungs and brain) has peaked scientific interest in NO $[41,42]$. The demonstration that NO plays a pivotal role in the control of several functions in the CNS (nociception, toxicity, degeneration, memory) has prompted the use of NO donors as probes to study NO in a variety of neurological diseases [43-46].

Among NO donors, nitroglycerin (NTG) has undergone extensive experimental investigation, because typical headaches develop in migraineurs (but not normals) with a 4-6 h latency after its administration [47-50]. NTG is highly lipophilic and easily crosses the BBB [42]. Experimental evidence for its accumulation in brain has been provided [51]. Systemic administration of this organic nitrate induces neuronal activation in several brain nuclei belonging to the neurovegetative, neuroendocrine, behavioural and nociceptive systems $[52,53]$. This activation develops with a latency of hours, which contrasts with the very short plasma half-life of NTG. Co-localisation studies show that NTG-induced neuronal activation takes place in adrenergic, nitrergic and neuropeptidergic structures [54, 55], thus suggesting some putative signalling pathways implicated in this model.

Exogenous (NTG-derived) NO might directly act at both the vascular and neuronal levels and indirectly evoke neurovascular responses via multiple pathways that include the synthesis of NO synthase and perhaps cyclooxygenase, and the induction of a trigeminovascularmediated biochemical response [56]. Neuronal activation following NTG shows that neuronal activation begins as early as $60 \mathrm{~min}$ postinjection in brain areas that control the cardiovascular function, and reaches a maximum $3 \mathrm{~h}$ later in nociceptive and related structures [52]. This modulated 
temporal course again suggests a dual mechanism of action for NTG; an initial effect on the vascular compartment followed by the involvement of integrative nociceptive structures. NTG administration evokes changes in the noradrenergic system at both the vascular and neuronal levels, and in the serotonergic system in specific brain areas [57]. Changes in central and/or peripheral neurotransmission may cause hyperalgesia, and a sustained activation of nociceptive nuclei in the rat [52]. In predisposed humans, this might initiate a spontaneous migraine attack following NTG administration [47-50]. This hypothesis has also been supported by other findings. Pardutz et al. [58] showed that NTG administration increases the number of NOS-immunoreactive cells in the rat spinal trigeminal nucleus, which points to the activation of secondorder neurones via a presynaptic excitatory mechanism. Lambert et al. [59] reported that systemic NTG increases the firing rate of second order trigeminal neurones, which transport inputs from cranial structures via a 5-HT-mediated mechanism that is prevented by the administration of selective 5-HT agonists. A valid criticism for many of these studies though is the use of large NTG dosages. Using doses more in line with human studies, Reuter et al. [60] demonstrated that NTG administration up-regulates pro-inflammatory genes such as iNOS, interleukin-6, interleukin-1, plus mast cell and macrophage activation, with a subsequent, delayed inflammatory reaction in the rat dura mater (4-6 h). Taken together, these findings strongly suggest that the study of NTG might lead to a better understanding of the pathophysiology of migraine attacks and of the role played by NO. Interestingly, SUM reduces CGRP levels and migraine pain during NTGinduced migraine attack [61].

To summarise, the experimental NI model represents a simple procedure to obtain preliminary information on well characterised receptor-targeted drugs. The apparent paradox observed with certain drugs that are shown to be effective in this model but not in clinical trials offers the opportunity to better manipulate structure-activity to obtain the best pharmacological profile using an array of experimental models.

\section{The migraine aura: ascending to the central origin of migraine}

Migraine is a common and debilitating disorder. Visual aura heralds the attacks in $20 \%$ of cases [62]. The typical aura is represented by an arc of scintillating, shining, crenellated shapes, beginning adjacent to central vision and expanding peripherally over 5-20 min, within one visual field, usually followed by headache. The scintillations are followed temporarily by a blind region, after the same retinotopic progression from central to peripheral visual fields. A relationship between cortical spreading depression (CSD) and migraine aura was first suggested by Leao [63]. CSD is a wave of neuronal and glial depolarisation, followed by long-lasting suppression of neural activity, and it can be evoked in mammals with lissencephalic $[63,64]$ or folded cortex [65]. Human neuroimaging such as planar Xenon [66-69], single photon emission tomography [68, 70-74] positron-emission tomography $[75,76]$, magnetoencephalography [77, 78] and MRI [79-81] support the hypothesis that CSD underlies migraine [82]. However many subjects never experience symptoms of typical visual auras in studies showing spreading hypoperfusion [75] or blood oxygenation level-dependent (BOLD) signal changes [79], and the initial hyperaemia characteristics of CSD were not directly demonstrated in human cortex.

High-field strength functional MRI to map progression of the BOLD events during migraine aura confirmed previous reports that CSD-like phenomena can be seen with neuroimaging techniques [80]. A slowly spreading area of perturbed BOLD signal has also been described in the occipital lobe during migraine aura. This perturbation shows an initial increase, perhaps corresponding to hyperaemia, followed by a decrease, and prolonged suppression of the light-evoked response. At least 8 features of CSD in the rat were found in these human studies. Based upon such findings and those of Cao [79], it seems highly likely that migraine aura is not evoked by ischaemia. More likely, it is evoked by aberrant firing of neurons and related cellular elements characteristic of CSD, and vascular changes develop due to fluctuations in neuronal activity during visual aura [83]. Drugs that inhibit the development and propagation of CSD provide novel treatment targets for both migraine aura, even before headache onset, as well as for stroke.

As discussed above, the trigeminal nerve innervates the meninges and participates in the genesis of migraine pain. However, the mechanisms that trigger migraine attacks are poorly understood. To better clarify this issue, recent data demonstrate that in the rat CSD activates trigeminovascular afferents and induces a series of cortical meningeal and brainstem events consistent with the development of headache [84]. CSD, in fact, is responsible for long-lasting blood-flow enhancement selectively within the middle meningeal artery upon activation of trigeminal and parasympathetic systems. Plasma protein leakage also occurs following CSD, within the dura mater in part by a neurokinin-1-receptor mechanism. These findings provide a neural mechanism by which extracerebral cephalic blood flow couples to brain events; this mechanism explains vasodilation during headache (including cluster headache) and links intense neurometabolic brain activity with the transmission of headache pain by the 
trigeminal nerve. Recently, CSD was shown to activate matrix metalloproteases with attendant albeit small changes in vascular permeability that were enduring and perhaps enable trigeminovascular activation [85]. It is possible that visual, motor or sensory aura might be responsible for the generation of the pain through the above mechanisms. In migraine without aura, the possibility is that CSD/aura may develop in "asymptomatic" or silent brain areas, to generate pain, independently from clinically evident focal symptoms.

\section{References}

1. Kellman DM (2002) The neuronal channelopathies. Brain 125:1177-1195

2. Moskowitz MA (1991) The visceral organ brain: implications for the pathophysiology of vascular head pain. Neurology 41:182-186

3. Moskowitz MA (1984) The neurobiology of vascular head pain. Ann Neurol 16:157-168

4. Saito K, Markowitz M, Moskowitz MA (1988) Ergot alkaloids block neurogenic extravasation in dura mater: proposed action in vascular headache. Ann Neurol 24:732-737

5. Markowitz S, Saito K, Moskowitz MA (1987) Neurogenically mediated leakage of plasma protein occurs from blood vessels in dura mater but not brain. J Neurosci 7:4129-4136

6. Buzzi MG, Moskowitz MA (1990) The antimigraine drug, sumatriptan (GR43175), selectively blocks neurogenic plasma extravasation from blood vessels in dura mater. Br J Pharmacol 99:202-206

7. Dimitriadou V, Buzzi MG, Moskowitz MA, Theoharides TC (1991) Trigeminal sensory fiber stimulation induces morphological changes reflecting secretion in rat dura mater mast cells. Neuroscience 44:97-112

8. Buzzi MG, Carter WB, Shimizu T, Heath H III, Moskowitz MA (1991) Dihydroergotamine and sumatriptan attenuate levels of CGRP in plasma in rat superior sagittal sinus during electrical stimulation of the trigeminal ganglion. Neuropharmacology 30:1193-1200

9. Buzzi MG, Dimitriadou V, Theoharides TC, Moskowitz MA (1992) 5Hydroxytryptamine receptor agonists for the abortive treatment of vascular headaches block mast cell, endothelial and platelet activation within the rat dura mater after trigeminal stimulation. Brain Res 583:137-149
10. Moskowitz MA, Buzzi MG (1991) Neuroeffector functions of sensory fibers: implications for headache mechanisms and drug action. J Neurol 238:S18-S22

11. Buzzi MG, Bonamini M, Moskowitz MA (1995) Neurogenic model of migraine. Cephalalgia 15:277-280

12. Kaube H, Hoskin HL, Goadsby PJ (993) Sumatriptan inhibits central trigeminal neurons only after bloodbrain barrier disruption. Br J Pharmacol 109:788-792

13. Goadsby PJ, Edvinsson L (1993) The trigeminovascular system and migraine: studies characterizing cerebrovascular and neuropeptide changes seen in humans and cats. Ann Neurol 33:48-56

14. Durham PL (2004) CGRP receptor antagonists: a new choice for acute treatment of migraine? Curr Opin Investig Drugs 5:731-735

15. Barbanti P, Fabbrini G, Pesare M, Vanacore N, Cerbo R (2002) Unilateral cranial autonomic symptoms in migraine. Cephalalgia 22:256-259

16. Barbanti P, Fabbrini G, Vanacore V, Pesare M, Buzzi MG (2003) Sumatriptan in migraine with unilateral cranial autonomic symptoms: an open study. Headache 43:400-403

17. Nissila M, Parkkola R, Sonninen P, Salonen R (1996) Intracerebral arteries and gadolinium enhancement in migraine without aura. Cephalalgia 16:363 (Abstract)

18. Pappagallo M, Szabo Z, Esposito G, Lakesh A, Velez L (1999) Imaging neurogenic inflammation in patients with migraine headache. Neurology 52[Suppl 2]:A274-A275

19. Gobel H, Czech N, Heinze-Kuhn K et al (2000) Evidence of regional protein plasma extravasation in cluster headache using Tc-99m albumin SPECT. Cephalalgia 20:287
20. Knight YE, Edvinsson L, Goadsby PJ (1999) Blockade of calcitonin generelated peptide release after superior sagittal sinus stimulation in cat a comparison of avitriptan and CP122,288. Neuropeptides 33:41-46

21. Lee WS, Moskowitz MA (1993) Conformationally restricted sumatriptan analogues, CP-122,288 and CP122,638 exhibit enhanced potency against neurogenic inflammation in dura mater. Brain Res 626:303-305

22. Roon KI, Olesen J, Diener HC et al (2000) No acute antimigraine efficacy of CP-122,288, a highly potent inhibitor of neurogenic inflammation: results of two randomized, doubleblind, placebo controlled clinical trials. Ann Neurol 47:238-241

23. Weiller C, May A, Limmroth V et al (1995) Brain stem activation in spontaneous human migraine attacks. Nat Med 1:658-660

24. Goadsby PJ, Hoskin KL (1998) Serotonin inhibits trigeminal nucleus activity evoked by craniovascular stimulation through a $5 \mathrm{HT} 1 \mathrm{~B} / 1 \mathrm{D}$ receptor: a central action in migraine. Ann Neurol 43:711-718

25. Storer RJ, Goadsby PJ (1997) Microiontophoretic application of serotonin (5HT)1B/1D agonists inhibits trigeminal cell firing in the cat. Brain 120:2171-2177

26. Goadsby PJ, Hoskin KL (1999) Differential effects of low dose CP122,288 and eletriptan on fos expression due to stimulation of the superior sagittal sinus in cat. Pain 82:15-22

27. Moskowitz MA, Macfarlane R (1993) Neurovascular and molecular mechanisms in migraine headaches. Cerebrovasc Brain Metab Rev 5:159-177 
28. Moskowitz MA, Nozaki K, Kraig RP (1993) Neocortical spreading depression provokes the expression of c-fos protein-like immunoreactivity within trigeminal nucleus caudalis via trigeminovascular mechanism. J Neurosci 13:1167-1177

29. Levy D, Jakubowski M, Burstein R (2004) Disruption of communication between peripheral and central trigeminovascular neurons mediates the antimigraine action of 5HT 1B/1D receptor agonists. Proc Natl Acad Sci USA 101:4274-4279

30. Garcia G, Kaufman MB, Colucci RD (1994) Dystonic reaction associated with sumatriptan. Ann Pharmacother 28:1199

31. Lopez-Alemany M, Ferrer-Tuset C, Bernacer-Alpera B (1997) Akathisia and acute dystonia induced by sumatriptan. J Neurol 224:131-132

32. Oterino A, Pascual J (1998) Sumatriptan-induced axial dystonia in a patient with cluster headache. Cephalalgia 18:360-361

33. Scott BL, Evans LW, Jankovic J (1996) Treatment of palatal myoclonus with sumatriptan. Mov Disord 11:748-751

34. Pascual J, Del Arco C, Romon T, Del Olmo E, Castro E, Pazos A (1996) Autoradiographic distribution of $[3 \mathrm{H}]$ sumatriptan-binding sites in postmortem human brain. Cephalalgia $16: 317-322$

35. Castro ME, Pascual J, Romon T, Del Arco C, Del Olmo E, Pazos A (1997) Differential distribution of [3] sumatriptan binding sites (5-HT1B, 5-HT1D and 5-HT1F receptors) in human brain: focus on brainstem and spinal cord. Neuropharmacology 36:535-542

36. Stepien A, Chalimoniuk M, Strosznajder J (1999) Serotonin 5HT1B/1D receptor agonists abolish NMDA receptor-evoked enhancement of nitric oxide synthase activity and Cgmp concentration in brain cortex slices. Cephalalgia 19:859-865

37. Lee WS, Limmroth V, Ayata C, Cutrer FM, Waeber C, Yu X et al (1995) Peripheral GABA A receptor-mediated effects of sodium valproate on dural plasma protein extravasation to substance $\mathrm{P}$ and trigeminal stimulation. $\mathrm{Br}$ J Pharmacol 116:1661-1667
38. Cutrer FM, Limmroth V, Ayata G, Moskowitz MA (1995) Attenuation by valproate of c-fos immunoreactivity in trigeminal nucleus caudalis induced by intracisternal capsaicin. Br J Pharmacol 116:3199-3204

39. Sandrini G, Milanov I, Proietti Cecchini A, Tassorelli C, Buzzi MG, Nappi G (2002) Electrophysiological evidence for trigeminal neuron sensitization in migraine. Neurosci Lett 317:135-138

40. Burstein R (2001) Deconstructing migraine headache into peripheral and central sensitization. Pain 89:107-110

41. Ignarro LJ (1991) Pharmacology of endothelium-derived nitric oxide and nitrovasodilators. West J Med 154:51-62

42. Murad F (1990) Drugs used for the treatment of angina: organic nitrates, calcium-channel blockers, and betaadrenergic antagonists. In: Goodman Gilman A, Rall TW, Nies AS, Taylor P (eds) Goodman and Gilman's the pharmacological basis of therapeutics. Raven Press, New York, pp 764-783

43. Meller ST, Gebhart GF (1993) Nitric oxide (NO) and nociceptive processing in the spinal cord. Pain 52:127-136

44. Kitto KF, Haley JE, Wilcox GL (1992) Involvement of nitric oxide in spinallymediated hyperalgesia in the mouse. Neurosci Lett 148:1-5

45. Urban MO, Gebhart GF (1999) Supraspinal contribution to hyperalgesia. Proc Natl Acad Sci USA 96:7687-7692

46. Aley KO, McCarter G, Levine JD (1998) Nitric oxide signaling in pain and nociceptor sensitization in the rat. J Neurosci 18:7008-7014

47. Iversen IK, Olesen J, Tfelt-Hansen P (1989) Intravenous nitroglycerin as an experimental model of vascular headache. Pain 38:17-24

48. Sicuteri F, del Bene ED, Poggioni M, Bonnazzi A (1987) Unmasking latent dysnociception in healthy subjects. Headache 27:180-185

49. Iversen HK, Olesen J (1994) Nitroglycerin-induced headache is not dependent on histamine release: support for a direct nociceptive action of nitric oxide. Cephalalgia 14:437-442

50. Olesen J, Thomsen LL, Lassen LH, Olesen IJ (1995) The nitric oxide hypothesis of migraine and other vascular headaches. Cephalalgia 5:94-100
51. Torfgard K, Ahnler J (1991) Tissue levels of glyceryl trinitrate and cGMP after in vivo administration in rat, and the effect of tolerance development. Can J Physiol Pharmacol 69:1257-1261

52. Tassorelli C, Joseph SA (1995) Systemic nitroglycerin induces Fos immunoreactivity in brainstem and forebrain structures of the rat. Brain Res 682:167-178

53. Tassorelli C, Joseph SA, Buzzi MG, Nappi G (1999) The effect on the central nervous system of nitroglycerin putative mechanisms and mediators. Progr Neurobiol 57:607-624

54. Tassorelli C, Joseph SA (1995) NADPHdiaphorase activity and Fos expression in brain nuclei following nitroglycerin administration. Brain Res 695:37-44

55. Tassorelli C, Joseph SA (1996) Systemic nitroglycerin activates peptidergic and catecholaminergic pathways in the rat brain. Peptides 17:443-449

56. Tassorelli C, Joseph SA, Nappi G (1997) Neurochemical mechanisms of nitroglycerin-induced neuronal activation in rat brain: a pharmacological investigation. Neuropharmacology 10:1417-1424

57. Tassorelli C, Blandini F, Costa A, Preza E, Nappi G (2002) Nitroglycerininduced activation of monoaminergic transmission in the rat. Cephalalgia 22:226-232

58. Pardutz A, Krizbai I, Multon S, Vecsei L, Schoenen J (2000) Systemic nitroglycerin increase nNOS levels in rat trigeminal nucleus caudalis.

Neuroreport 11:3071-3075

59. Lambert GA, Donaldson C, Boers PM, Zagami AS (2000) Activation of trigeminovascular neurons by glyceryl trinitrate. Brain Res 887:203-210

60. Reuter U, Bolay H, Jansen-Olesen I, Chiarugi A, Sanchez del Rio M, Letourneau R et al (2001) Delayed inflammation in rat meninges: implications for migraine pathophysiology. Brain 124:2490-2502

61. Juhasz G, Zsombok T, Jakab B, Nemeth J, Szolcsanyi J, Bagdy G (2005) Sumatriptan causes parallel decrease in plasma calcitonin generelated peptide (CGRP) concentration and migraine headache during nitroglycerin induced migraine attack. Cephalalgia 25:179-183 
62. Russell MB, Olesen J (1996) A nosographic analysis of the migraine aura in a general population. Brain 119:355-361

63. Leao AA (1944) Spreading depression of activity in cerebral cortex. $\mathbf{J}$ Neurophysiol 7:359-390

64. Leao AA (1986) Spreading depression. Funct Neurol 1:363-366

65. James MF, Smith MI, Bockhorst KH, Hall LD, Houston GC, Papadakis NG, Smith JM, Williams AJ, Xing D, Parsons AA et al (1999) Cortical spreading depression in the gyrencephalic feline brain studied by magnetic resonance imaging. J Physiol 519:415-425

66. Lauritzen M, Skyhoj Olsen T, Lassen NA, Paulson OB (1983) Changes in regional cerebral blood flow during the course of classic migraine attacks. Ann Neurol 13:633-641

67. Lauritzen M, Olesen J (1984) Regional cerebral blood flow during migraine attacks by Xenon-133 inhalation and emission tomography. Brain 107:447-461

68. Olesen J, Lauritzen M, Tfelt-Hansen P, Henriksen L, Larsen B (1982) Spreading cerebral oligemia in classical and normal cerebral blood flow in common migraine. Headache 22:242-248

69. Skyhoj Olsen T, Friberg L, Lassen NA (1987) Ischemia may be the primary cause of the neurologic deficits in classic migraine. Arch Neurol 44:156-161

70. Andersen AR, Friberg L, Olsen T, Olesen J (1988) Delayed hyperemia following hypoperfusion in classic migraine. Single photon emission computed tomographic demonstration. Arch Neurol 45:154-159
71. La Spina I, Vignati A, Porazzi D (1997) Basilar artery migraine: transcranial Doppler EEG and SPECT from the aura phase to the end. Headache 37:43-47

72. Olesen J, Friberg L, Skyhoj Olsen T, Iversen HK, Lassen NA, Andersen AR, Karle A (1990) Timing and topography of cerebral blood flow, aura, and headache during migraine attacks. Ann Neurol 28:791-798

73. Seto H, Shimizu M, Futatsuya R, Kageyama M, Wu Y, Kamei T, Shibata R, Kakishita M (1994) Basilar artery migraine. Reversible ischemia demonstrated by Tc-99m HMPAO brain SPECT. Clin Nucl Med 19:215-218

74. Soriani S, Feggi L, Battistella PA, Arnaldi C, De Carlo L, Stipa S (1997) Interictal and ictal phase study with Tc 99m HMPAO brain SPECT in juvenile migraine with aura. Headache 37:31-36

75. Woods RP, Iacoboni M, Mazziotta JC (1994) Brief report: bilateral spreading cerebral hypoperfusion during spontaneous migraine headache. $\mathrm{N}$ Engl J Med 331:1689-1692

76. Andersson JL, Muhr C, Lilja A, Valind S, Lundberg PO, Langstrom B (1997) Regional cerebral blood flow and oxygen metabolism during migraine with and without aura. Cephalalgia 17:570-579

77. Tepley N, Wijesinghe RS (1996) A dipole model for spreading cortical depression. Brain Topogr 8:345-353

78. Barkley GL, Tepley N, Simkins R, Moran J, Welch KM (1990) Neuromagnetic fields in migraine: preliminary findings. Cephalalgia 10:171-176
79. Cao Y, Welch KM, Aurora S, Vikingstad EM (1999) Functional MRI-BOLD of visually triggered headache in patients with migraine. Arch Neurol 56:548-554

80. Cutrer FM, Sorensen AG, Weisskoff RM, Ostergaard L, Sanchez del Rio M, Lee EJ, Rosen BR, Moskowitz MA (1998) Perfusion-weighted imaging defects during spontaneous migrainous aura. Ann Neurol 43:25-31

81. Sanchez del Rio M, Bakker D, Wu O, Agosti R, Mitsikostas DD Ostergaard L, Wells WA, Rosen BR, Sorensen G, Moskowitz MA, Cutrer FM (1999) Perfusion weighted imaging during migraine: spontaneous visual aura and headache. Cephalalgia 19:701-707

82. Lauritzen M (2000) Cortical spreading depression. In: Olesen J, Tfelt-Hansen P, Welch KMA (eds) The headaches, 2nd edn. Lippincott, Philadelphia, PA, pp 189-194

83. Hadjikhani N, Sanchez del Rio M, Wu O, Schwartz D, Bakker D, Fischl B, Kwong KK, Cutrer FM, Rosen BR, Tootell RBH, Sorensen AG, Moskowitz MA (2001) Mechanisms of migraine aura revealed by functional MRI in human visual cortex Proc Natl Acad Sci USA 98:4687-4692

84. Bolay H, Reuter U, Dunn AK, Huang Z, Boas DA, Moskowitz MA (2002) Intrinsic brain activity triggers trigeminal meningeal afferents in a migraine model. Nat Med 8:136-142

85. Gursoy-Ozdemir Y, Qiu J, Matsuoka N, Bolay H, Bermpohl D, Jin H, Wang X, Rosenberg GA, Lo EH, Moskowitz MA (2004) Cortical spreading depression activates and upregulates M MP-9. J Clin Invest 113:1447-1455 